\title{
Ability of a commercial feed additive to modulate expression of innate immunity in sheep immunosuppressed with dexamethasone
}

\author{
Y. Wang ${ }^{1}$, S. B. Puntenney ${ }^{1}$, J. L. Burton ${ }^{2}$ and N. E. Forsberg ${ }^{1+}$ \\ ${ }^{1}$ Department of Animal Sciences, Oregon State University, Corvallis, OR 97331-6702, USA; ${ }^{2}$ Department of Animal Science, Immunogenetics Laboratory and \\ Center for Animal Functional Genomics, Michigan State University, East Lansing, MI 48824, USA
}

(Received 26 September 2006; Accepted 10 May 2007)

\begin{abstract}
In the first study, we tested the ability of a commercial feed additive (OmniGen-AF) to affect markers of innate immunity in immunosuppressed sheep and the ability of a pathogen challenge (mould) to affect the immune response to the additive. Treatments consisted of (1) control, (2) immunosuppressed with dexamethasone (DEX), (3) immunosuppressed plus the feed additive, (4) immunosuppressed plus Aspergillus fumigatus and (5) immunosuppressed, A. fumigatus and the additive. Animal health was monitored and indexes of innate immunity (neutrophil L-selectin and interleukin-1 $\beta$ (IL-1 $\beta)$ ) were collected. DEX caused immunosuppression (i.e. reduced abundance of neutrophil L-selectin and IL-1 $\beta$ ). This immunosuppressive effect was countered by the provision of the additive in the ration. Provision of mould in the ration increased the ability of the additive to regulate markers of innate immune function. A second study was completed to re-assess the properties of the additive and other feed products. The study consisted of seven treatments: (1) immunosuppressed, (2) immunosuppressed with additive, (3) immunosuppressed with additive in pelleted form (low-temperature pellet) and (4) immunosuppressed with additive in a high-temperature pellet. The remaining three treatments assessed abilities of three other additives to regulate markers of innate immune function. In this study, OmniGen-AF increased expression of neutrophil L-selectin abundance in immunosuppressed animals and this was unaffected by the pelleting temperature. None of the other additives affected markers of innate immunity. In these studies we discovered mechanisms by which a feed product may affect the immune function of ruminant livestock. The product countered DEX-dependent down-regulation of markers of innate immune function and its actions were enhanced by the presence of pathogen (mould) in the ration.
\end{abstract}

Keywords: Aspergillus fumigatus, immunity, interleukin-1 $\beta$, L-selectin, stress

\section{Introduction}

The immune system consists of a wide spectrum of interacting systems that are designed to minimise the likelihood of an infection. The first line of defence of the immune system has been termed 'innate immunity' and consists of epithelial barriers, complement, digestive acid and enzymes and a cellular component (e.g. neutrophils, macrophages). The adaptive system is termed the 'antibody system' and is mediated by the expression of antigen-specific antibodies by $B$ and $T$ lymphocytes. Development of an antibody response requires time for development; hence, animals rely upon their innate immune system to prevent an infection while the antibodies specific to the pathogen challenge are developing (Janeway et al., 2005).

${ }^{\dagger}$ E-mail: neil.forsberg@orst.edu
The term 'innate' immunity implies that it is an unwavering or stable aspect of the immune system. However, there are considerable differences from animal to animal in the 'strength' of the innate immune system (Janeway et al., 2005). The degree of stress that an animal experiences is an important determinant of the extent to which its innate immune system may be compromised. Stress is an important immunosuppressive event and, in addition to downregulating the adaptive immune system, reduces expression of key mediators of innate immunity. The stress-related down-regulation of both the innate and adaptive immune responses of livestock is responsible, to a considerable extent, for the abilities of pathogens to cause a systemic, possibly fatal, infection.

Dairy cattle are immunosuppressed during various stages of lactation (e.g. at freshening and at dry-off) when isolated in a 'hospital pen' and when exposed to extreme heat 
(Mallard et al., 1998; Madsen et al., 2004; Burton et al., 2005). These periods of immunosuppression correspond to a higher incidence of disease (Burton and Erskine, 2003). To address the problems associated with immunosuppression in dairy cattle, a feed supplement (OmniGen-AF) is commonly used in the dairy industry. In these experiments, we assessed the effects this feed additive exerted on markers of innate immunity. Effects of the additive were examined in the absence and in the presence of a pathogen challenge (Aspergillus fumigatus-infected silage) because field studies have indicated that the product may have more efficacy in the presence of a pathogen challenge. Our hypothesis was that this additive would increase expression of markers of neutrophil function in the presence and absence of a pathogen challenge.

\section{Material and methods}

All research reported was approved by Oregon State University's Institutional Animal Care and Use Committee.

\section{Experiment 1}

Objectives of the first study were to assess the effects of a feed additive (OmniGen-AF) on expression of markers of innate immunity in neutrophils of immunosuppressed sheep. To carry out this experiment, 60 Polypay $\times$ Friesan/ Suffolk sheep (25 wethers and 35 ewe lambs: ca. $37.5 \mathrm{~kg}$ each) were assigned to five treatments. Five males and seven females were randomly assigned to each treatment. The duration of the experiment was 28 days.

The treatments consisted of: (1) control, (2) immunosuppressed, (3) immunosuppressed and supplemented with the feed additive, (4) immunosuppressed and pathogenchallenged and (5) immunosuppressed, pathogen-challenged and supplemented with the feed additive.

Immunosuppression was induced by a twice daily injection of dexamethasone (DEX) (Azium: $0.1 \mathrm{mg} / \mathrm{kg}$ body weight, twice a day). This is a well-established model of stress-induced immunosuppression in ruminants, which was reported by Dr Jeanne Burton at Michigan State University (Burton and Kehrli, 1995; Burton et al., 1995; Weber et al., 2001). Azium was injected subcutaneously in the neck region. The feed additive used was OmniGen-AF ${ }^{\circledR}$ (PrinceAgri Products, Quincy, IL, USA). This was fed to animals in treatments 3 and 5 at a dose of $0.5 \%$ of a concentrate supplement $(\mathrm{w} / \mathrm{w})$ and provided $4 \mathrm{~g}$ per head per day. Pathogen challenge was provided to treatments 4 and 5 by daily feeding of highly moulded pelleted wheat mill run $(0.45 \mathrm{~kg}$ per head per day). This sample of wheat mill run had been obtained from a dairy in Washington State with high incidence of a gastro-intestinal (GI) infection (haemorrhagic bowel syndrome) and abortions. In lieu of the mouldy mill run, treatments 1,2 and 3 were provided wheat bran $(0.45 \mathrm{~kg}$ per head per day). All animals were fed alfalfa hay free choice and provided $0.34 \mathrm{~kg}$ of ground maize per head per day. Sources of ground maize and wheat bran were baked at $95^{\circ} \mathrm{C}$ for $24 \mathrm{~h}$ prior to feeding to reduce the likelihood of introducing viable extraneous pathogens to animals on treatments 1 , 2 and 3.

Animal health. Animal health was monitored throughout the trial. Rectal temperatures were taken weekly. When animals appeared depressed, a veterinarian from the Oregon State University College of Veterinary Medicine (OSUCVM) was employed to diagnose illnesses. Two animals that exhibited elevated body temperatures at the end of the trial were euthanased by electrocution at the OSUCVM by a licensed veterinary pathologist. Necropsies were performed on both animals with particular attention to GI pathologies. Faecal consistency was also monitored subjectively throughout the trial as a means of assessing whether the treatments induced $\mathrm{Gl}$ anomalies. Faecal consistency scores were assigned at weighing. A score of ' 0 ' was assigned to animals with solid, normal faeces. A graded score of ' 1 ' to ' 3 ' was assigned to animals with scouring ranging from mild, to moderate to severe, respectively. Animals were weighed weekly.

Following completion of this study, animals were held in the Oregon State University sheep facility for a 28-day clearance period. This was judged by the OSUCVM to provide adequate time for clearance of residual DEX.

Isolation of neutrophils. A $10 \mathrm{ml}$ blood sample was taken via jugular puncture on the 28th day of the study. Blood was transferred into $50 \mathrm{ml}$ tubes containing $5 \mathrm{ml}$ of acid citrate dextrose anti-coagulant. Tubes were centrifuged at $1000 \times \mathbf{g}$ for $20 \mathrm{~min}$ at $4^{\circ} \mathrm{C}$ in a swinging bucket centrifuge to separate plasma and buffy coats from the red cell pack. Plasma, buffy coat and two-thirds of the red cell pack from each tube were aspirated aseptically and discarded. The remaining red cell packs were mixed with $20 \mathrm{ml}$ of ice-cold phosphate-buffered saline (PBS). Samples were divided into five tubes $(50 \mathrm{ml}$ Falcon, BD), and $30 \mathrm{ml}$ of ice-cold PBS were added and under-layered with $10 \mathrm{ml}$ of $1.084 \mathrm{~g} / \mathrm{ml}$ Percoll (Sigma Chemical Company, St Louis, MO, USA), then centrifuged at $400 \times \mathbf{g}$ for $40 \mathrm{~min}$ at $22^{\circ} \mathrm{C}$. After centrifugation, the erythrocytes and neutrophils pelleted at the bottom of the tube and the mononuclear cells remained at the sample/medium interface (band). Supernatant, mononuclear cell layer and Percoll were aspirated aseptically. Erythrocytes were lysed using $12 \mathrm{ml}$ ice-cold hypotonic lysing solution $\left(10.56 \mathrm{mmol} / / \mathrm{Na}_{2} \mathrm{HPO}_{4}, 2.67 \mathrm{mmol} / \mathrm{l}\right.$ $\mathrm{NaH}_{2} \mathrm{PO}_{2}, \mathrm{pH}$ 7.3) for $90 \mathrm{~s}$. Isotonicity was restored by adding $6 \mathrm{ml}$ ice-cold hypertonic restoring solution $\left(10.56 \mathrm{mmol} / \mathrm{l} \mathrm{Na} 2 \mathrm{HPO}_{4}, 2.67 \mathrm{mmol} / / \mathrm{NaH}_{2} \mathrm{PO}_{2}, 0.43 \mathrm{~mol} / \mathrm{l}\right.$ $\mathrm{NaCl}$, pH 7.3). Remaining leukocytes were pelleted by centrifugation at $800 \times \mathbf{g}$ for $5 \mathrm{~min}$ at $4^{\circ} \mathrm{C}$. These granu-

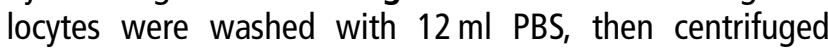
$800 \times \mathbf{g}$ for $5 \mathrm{~min}$ at $4^{\circ} \mathrm{C}$. Purity of the neutrophil preparations was examined with flow cytometry with purities of typical isolations being $>93 \%$ neutrophils. 
Western blot analyses. Whole-cell protein from neutrophils $(20 \mu \mathrm{g})$ was applied to SDS-PAGE gels from three randomly chosen wethers and three randomly chosen ewe lambs on each treatment and electrophoresed with molecular weight markers. Samples were then transferred to a nitrocellulose membrane via Western blotting (Brent et al., 2003) and membranes were blocked with 5\% skim milk in Tween-20, Tris-buffered saline (TTBS). Primary antibody (anti-bovine L-selectin or interluekin-1 $\beta$ (IL-1 $\beta$ ): VMRD, Pullman, WA, USA) was exposed to membranes for $1 \mathrm{~h}$ after which membranes were exposed to secondary antibody (goat antimouse-horseradish peroxidase: BioRad, Hercules, CA, USA) for $1 \mathrm{~h}$. Membranes were exposed to developer (BioRad ImmunStar chemiluminescent detection kit for $30 \mathrm{~s}$ ). Membranes were washed five times with TTBS and then exposed to an X-ray film for $1 \mathrm{~h}$ (L-selectin: $40 \mathrm{kDa}$ ) or overnight (IL-1 $\beta$ : $30 \mathrm{kDa}$ ) and developed. Intensity of exposures was determined by scanning densitometry using a BioRad VersaDoc 1000 imager and PDQuest software. Concentrations of L-selectin and IL-1 $\beta$, as a proportion of total cellular protein, were expressed in arbitrary densitometer units.

Quantitative Sybr-Green PCR. We tested feeds and a ruminal wall mycotic lesion for the presence of $A$. fumigatusspecific DNA using methods previously described by Puntenney et al. (2003). In all Sybr-Green assays, a standard curve of $A$. fumigatus genomic DNA was developed. $A$. fumigatus genomic DNA standard was obtained from the Scientific Institute of Public Health (Brussels, Belgium).

\section{Experiment 2}

Objectives of the second experiment were as follows: (1) to assess the ability of the feed additive to increase neutrophil L-selectin expression in immunosuppressed animals, (2) to determine whether this property of the additive was eliminated by its incorporation into pelleted feeds and (3) to examine abilities of three additional additives to regulate neutrophil L-selectin abundance. We also assessed effects of OmniGen-AF on blood cell numbers in immunosuppressed animals.

Forty-nine Polypay $\times$ Friesan/Suffolk sheep (28 wethers and 21 ewe lambs) were assigned to seven treatments such that each treatment contained four wethers and three ewe lambs. Animals were not used in prior experimentation (i.e. experiment 1). Wethers and ewes were randomly assigned to the seven treatments. Initial starting weight of the animals was approximately $45 \mathrm{~kg}$. Animals were weighed at the conclusion of the trial ( 28 days) and weight gains were calculated.

The seven treatments consisted of (1) immunosuppressed (as described in experiment 1), (2) immunosuppressed with supplementation of OmniGen-AF (3.8 g per head per day) in powdered form, (3) immunosuppressed with supplementation of OmniGen-AF (3.8 g per head per day) in a pellet formed by heating to $71^{\circ} \mathrm{C}$, (4) immunosuppressed with supplementation of OmniGen-AF ( $3.8 \mathrm{~g}$ per head per day) in a pellet heated to $82^{\circ} \mathrm{C}$, (5) immunosuppressed with supplementation of DTX ( $0.95 \mathrm{~g}$ per head per day), an additive available from Select Sires (Plain City, $\mathrm{OH}$ ), (6) immunosuppressed with supplementation of MTB-100 (1.2 g per head per day; Alltech, Nicholasville, KY, USA) and (7) immunosuppressed with supplementation of NutrasoundPak $(2.52 \mathrm{~g}$ per head per day; Archer Daniels Midland, Decatur, IL, USA). The two different pelleting temperatures reflect the range of temperatures used in commercial pelleting mills in the United States. All additives were included in the rations as recommended on feeding labels. Where sheep recommendations were not included, $10 \%$ of the dairy recommendations were utilised.

The ration consisted of free-choice forage and a concentrate mixture. The forage was a 50:50 mixture of chopped alfalfa and spoiled grass silage. Spoiled silage was prepared by sampling a bunker silo of grass silage from the OSU dairy. Silage was transferred to a truck and transported to the university sheep centre. During both loading and unloading, the silage was aerated and, as a result, mould growth was facilitated. The rationale for including the spoiled silage was that results from experiment 1 had indicated that the presence of a pathogen may potentiate actions of OmniGen-AF. The concentrate portion of the ration was provided at $0.45 \mathrm{~kg}$ per head per day. For treatments $1,2,5,6$ and 7 , this concentrate mixture was fed as a mash. Concentrate mixtures used for treatments 3 and 4 were pelleted at $71^{\circ} \mathrm{C}$ and $82^{\circ} \mathrm{C}$, respectively.

Blood samples were taken on the 28th day of the study and neutrophils prepared as outlined earlier. Neutrophil protein samples from wethers were applied to lanes 1 to 4 of SDS-PAGE gels and samples from ewe lambs were applied to lanes 5 to 7 . Additional blood samples from animals on treatments 1 and 2 were transported to the Veterinary Diagnostic Laboratory at the OSU Veterinary Diagnostic Laboratory for neutrophil and lymphocyte counting using a Baker 9000 hematology analyser (Irvine, (A, USA).

\section{Statistical analyses}

Effects of the five treatments (experiment 1) and the seven treatments (experiment 2) on all experimental variables were assessed by mixed model analysis using the ANOVA procedure of Statistical Analysis Systems Institute (2005) and Steel and Torrie (1980). The statistical model included the fixed effects of treatment and random animal and error effects. A Student-Neuman-Keul multiple range test was then used to test for individual treatment differences. A 5\% level of significance was adopted for all comparisons.

\section{Results}

Experiment 1

Average daily weight gains for animals on treatments 1 to 5 were $0.21,0.15,0.16,0.08$ and $0.15 \mathrm{~kg} /$ day, respectively. 
On weeks 2, 3 and 4 , animals on treatment 1 weighed significantly more $(P<0.05)$ than animals on the remaining four treatments. No differences $(P>0.05)$ in weights were detected among animals on treatments 2, 3, 4 and 5 although animals on treatment 4 (immunosuppressed and exposed to mouldy feed) declined in weight $(P>0.05)$ in week 4.

Significant differences in body temperatures were not detected $(P>0.05)$ among animals on the five treatments. However, in the fourth week of the study, three sheep on treatment 4 developed lethargy, ruminal hypomotility and pyrexia (each over $40^{\circ} \mathrm{C}$ ). The attending veterinarian diagnosed pneumonia in these animals. The two most severely affected of these three animals were euthanased in the OSUCVM by a veterinary pathologist following completion of the trial. One was devoid of any visible Gl pathology. The other demonstrated moderate but locally extensive ulcerative rumenitis with mild, emphysematous lymphadenopathy. In the centre of the ruminal lesion, we also found a blue/grey-coloured mycotic lesion that tested positive for $A$. fumigatus using quantitative Sybr-Green PCR. Associated lymph nodes also tested positive for A. fumigatus DNA.
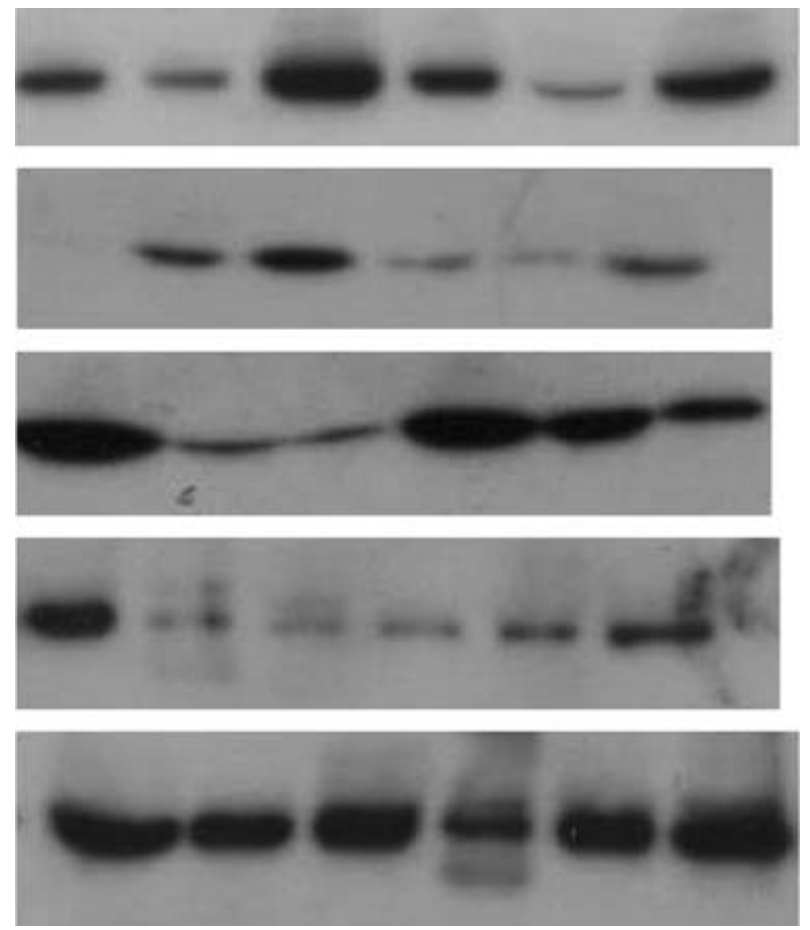

Figure 1 Effects of immunosuppression of the feed additive (OmniGenAF) and of mould on concentrations of L-selectin protein in neutrophils. Panel 1 (top) represents control animals. Animals in panel 2 were injected with dexamethasone (immunosuppressed). Animals in panel 3 were immunosuppressed and fed the additive. Animals in panel 4 were immunosuppressed and challenged with mouldy feed. Animals in panel 5 were immunosuppressed, fed the additive and challenged with mouldy feed. Six animals are represented in each panel. Size of the L-selectin protein is $40 \mathrm{kDa}$.
Faecal consistency scores were monitored throughout the study. Mean faecal scores on week 4 for treatments 1 to 5 were $1.25,0.17,0.33,0.17$ and 0.33 , respectively. DEX treatment caused a significant improvement $(P<0.05)$ in fecal consistency (treatments 2 to 5 compared with treatment 1). Differences among treatments 2 to 5 were not detected $(P>0.05)$.

Administration of DEX caused marked immunosuppression. Evidence of this is given in Figures 1 and 2 and in Table 1. DEX injection reduced neutrophil L-selectin abundance by $62 \%$ and IL- $1 \beta$ abundance by $99 \%$. Addition of the feed additive to the ration of immunosuppressed animals in treatment 3 increased $(P<0.05)$ L-selectin protein concentration by two-fold (compared with non-immunosuppressed controls) but had no effect $(P>0.05)$ on IL-1 $\beta$ concentration (Table 1).

Animals on treatments 4 and 5 were challenged with mouldy feed. We tested this feed source using quantitative PCR and determined that it was contaminated with A. fumigatus ( $>1$ million spores per $\mathrm{g}$ ). Provision of the mouldy feed source to immunosuppressed animals (treatment $4 v$. treatment 2) had no effect $(P>0.05)$ on the abundance of L-selectin or IL-1 $\beta$ in neutrophils.

Addition of the feed additive to immunosuppressed, mould-challenged sheep increased $(P<0.05)$ L-selectin abundance to levels that were similar to animals given the additive in treatment 3 (Figure 1, Table 1). Mouldy feed increased actions of the additive on neutrophil IL- $1 \beta$ protein abundance. Whereas the additive had no effect on IL-1 $\beta$ abundance in the absence of the mould challenge (treatment $3 v$. treatment 2), the additive increased IL-1 $\beta$ protein concentration by two-fold (compared with control-fed animals) when fed in the presence of the mould (treatment $5 \mathrm{v}$. treatment 4).

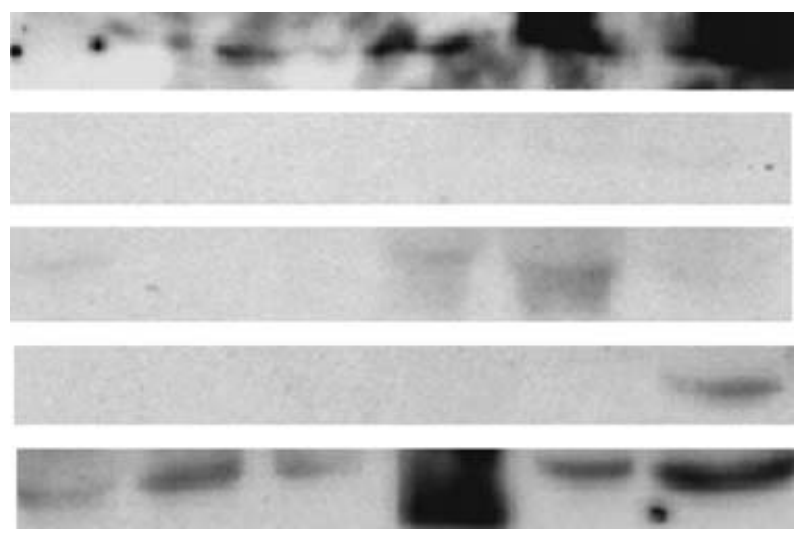

Figure 2 Effects of immunosuppression, of the feed additive (OmniGen$\mathrm{AF})$ and of mould on concentrations of interleukin- $1 \beta$ protein in neutrophils. Panel 1 (top) represents control animals. Animals in panel 2 were injected with dexamethasone (immunosuppressed). Animals in panel 3 were immunosuppressed and fed the additive. Animals in panel 4 were immunosuppressed and challenged with mouldy feed. Animals in panel 5 were immunosuppressed, fed the additive and challenged with mouldy feed. Six animals are represented in each panel. Size of the interleukin- $1 \beta$ protein is $30 \mathrm{kDa}$. 
Nutritional support of immunity in sheep

Table 1 Concentrations of L-selectin protein and interleukin-1 $\beta$ protein in neutrophils isolated form the five treatment groups in experiment $1^{+}$

Immunosuppressed +

Immunosuppressed Immunosuppressed+OmniGen-AF Immunosuppressed+mould mould+OmniGen-AF

\begin{tabular}{llllll}
\hline L-selectin & $127^{\mathrm{a}} \pm 24$ & $48^{\mathrm{b}} \pm 17$ & $206^{\mathrm{c}} \pm 59$ & $54^{\mathrm{b}} \pm 27$ & $295 \pm 92^{\mathrm{c}}$ \\
Interleukin-1 $\beta$ & $133^{\mathrm{a}} \pm 21$ & $1.3^{\mathrm{b}} \pm 1$ & $14^{\mathrm{b}} \pm 12$ & $23^{\mathrm{b}} \pm 23$ & $273 \pm 101^{\mathrm{c}}$ \\
\hline \hline
\end{tabular}

a,b,c Values within a row which do not share a common superscript differ significantly $(P<0.05)$.

${ }^{\dagger}$ Values are expressed in arbitrary densitometer units per milligram of total neutrophil protein. Values are given as means \pm s.e.m.

\section{Experiment 2}

Clinical signs of illness and lethargy were not observed in any of the immunosuppressed animals during the 28-day experiment. Body temperatures were not significantly elevated $(P>0.05)$ in any treatment. Weight gains of the animals on treatments 1 to 7 were $0.21,0.21,0.30,0.31$, $0.19,0.18$ and $0.24 \mathrm{~kg} /$ day, respectively, for the 28 -day trial. Animals on treatments 2, 5, 6 and 7 did not differ in weight gain from the control-fed animals (treatment $1 ; P>0.05$ ). However, animals that received pelleted diets (treatments 3 and 4) gained significantly more weight $(P<0.05)$ than animals on all other treatments. Animals on treatment 3 (low-temperature pellet) gained $43 \%$ more weight $(P<0.05)$ and animals on treatment 4 (high-temperature pellet) gained $47 \%$ more weight $(P<0.05)$ than control-fed animals.

All animals in this experiment were immunosuppressed via daily injection of DEX. Effects of the seven experimental treatments on the abundance of neutrophil L-selectin protein are shown in Figure 3. Very low levels of L-selectin protein were detected in control sheep (Figure 3, panel 1). Addition of OmniGen-AF increased the abundance of neutrophil L-selectin protein irrespective of whether it was fed as a mash or was pelleted (Figure 3, see panels 2, 3 and 4). Addition of DTX, MTB-100 or Nutrasound-Pak to the rations of immunosuppressed sheep had no effect $(P>0.05)$ on the abundance of neutrophil L-selectin (Figure 3, panels 5, 6 and 7, respectively).

Addition of OmniGen-AF to the rations of immunosuppressed sheep increased circulating concentrations of both neutrophils and lymphocytes $(P<0.05)$. Concentrations of neutrophils in control $v$. OmniGen-AF-fed sheep were $2814 \pm 401$ v. $4226 \pm 476$ cells per $\mu$ l, respectively. Concentrations of leukocytes in control v. OmniGen-AF-fed sheep were $4001 \pm 404 v$. $5923 \pm 511$ cells per $\mu$ l, respectively.

\section{Discussion}

\section{Experiment 1}

We tested a hypothesis that feeding OmniGen-AF to immunosuppressed sheep would increase abundance of markers of innate immunity in neutrophils. Results indicated that the product increased expression of innate immune markers (L-selectin and IL-1 $\beta$ ) in immunosuppressed sheep and that this effect was more pronounced in animals that were also exposed to a pathogen ( $A$. fumigatus) challenge.
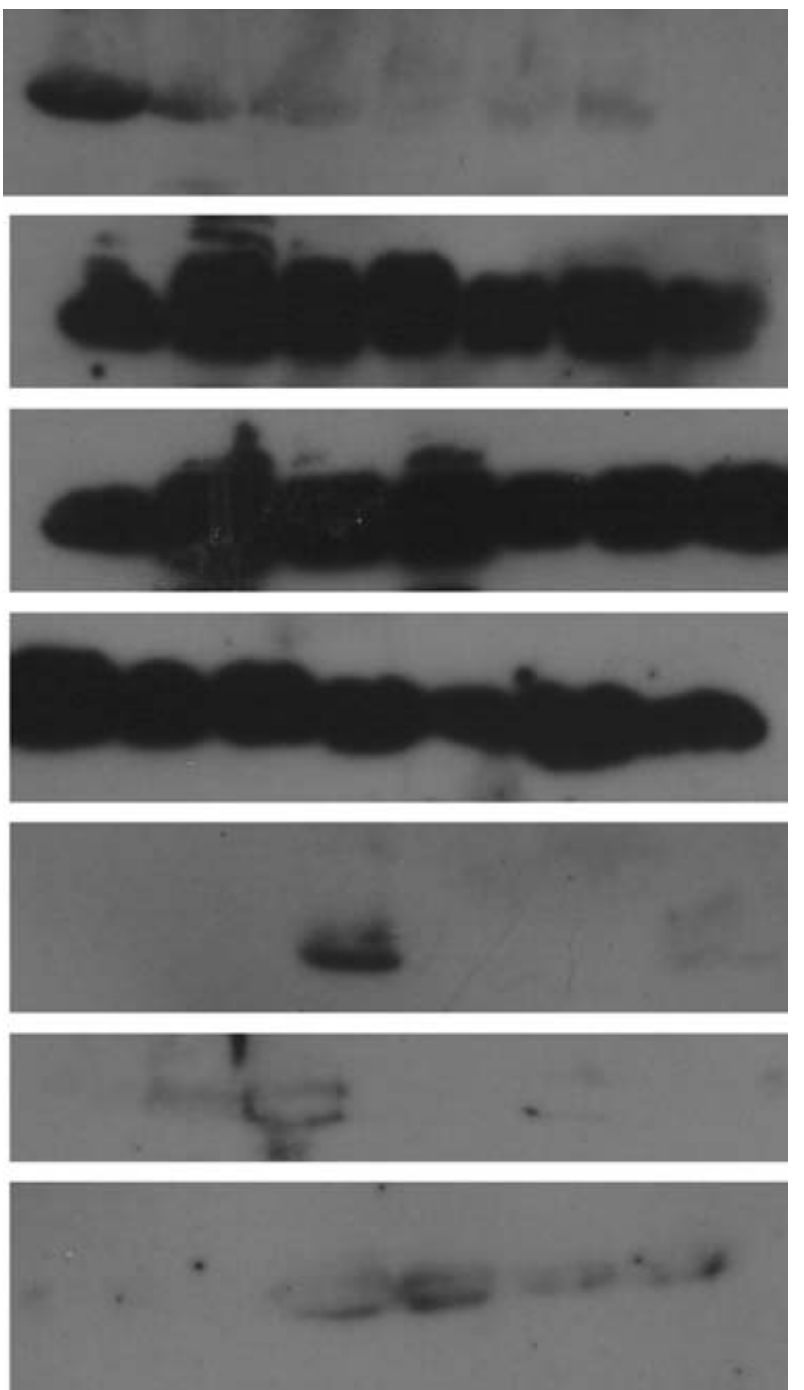

Figure 3 Effects of various feed additives and of pelleting on concentrations of neutrophil L-selectin in immunosuppressed sheep. Panel 1 (top) represents immunosuppressed control animals. Animals in panel 2 were fed OmniGen-AF in a mash form. Animals in panels 3 and 4 were fed OmniGen-AF in pelleted forms $\left(71\right.$ and $82^{\circ} \mathrm{C}$, respectively). Animals in panels 5, 6 and 7 were provided with DTX, MTB-100 and Nutrasound-Pak, respectively. Neutrophil L-selectin concentrations from seven animals are represented in each panel. Size of the L-selectin protein is $40 \mathrm{kDa}$.

L-selectin is an extracellular neutrophil adhesion molecule that enables the neutrophil to interact weakly with endothelial selectins and, thereby, to roll along the internal surface of blood vessels and 'search' for sites of infection 
(Weber et al., 2004). Weber et al. (2001) have reported that L-selectin is a 'plastic' reporter protein on neutrophils that is down-regulated at the mRNA and protein levels by glucocorticoids (Burton and Kehrli, 1995; Burton et al., 1995). Glucocorticoid-related immunosuppression of dairy cattle at parturition also is associated with the down-regulation of neutrophil L-selectin protein concentrations and this may explain, in part, the prevalence of infections associated with early lactation (Burton and Erskine, 2003; Burton et al., 2005).

In this study, administration of a glucocorticoid reduced neutrophil L-selectin abundance and this effect was completely reversed by the feeding of OmniGen-AF irrespective of feed mould status. Ability of OmniGen-AF to restore neutrophil L-selectin concentrations in immunosuppressed sheep implies that it has the potential of restoring the allimportant infection surveillance function of neutrophils and, possibly, to maintain innate immunity during times of stress.

Intriguingly, the feed product also restored glucocorticoid-induced down-regulation of IL-1 $\beta$. However, this occurred primarily when a mould challenge was also provided. IL-1 $\beta$ is a potent cytokine secreted by neutrophils (and other immune cells) when infection is first encountered. Among its various functions, IL- $1 \beta$ is key in activating the vascular endothelium and thereby causing it to become sticky for rolling neutrophils. Thus, IL-1 $\beta$ increases the ability of these phagocytic cells to gain quick access to sites of infections below the blood vessels (Janeway et al., 2005). IL-1 $\beta$ is also involved in the acute 'sickness response' of infected animals, inducing the brain to set off a stress response and the liver to increase its production of acute phase proteins that ultimately facilitate neutrophil uptake of the infecting pathogen. Furthermore, IL-1 $\beta$ provides an important linkage between the innate and adaptive arms of the immune system by providing an activating signal for lymphocyte proliferation (Janeway et al., 2005). Thus, restoration of IL-1 $\beta$ expression by the feed additive in otherwise DEX-immunosuppressed animals implies that the product may have the potential to restore innate immunity in other immunosuppressed livestock.

The mechanism(s) by which the additive restored expression of neutrophil L-selectin and IL-1 $\beta$ is not known. The product contains natural products of yeast and fungal organisms, in addition to other ingredients, which we propose account for some or all of the product's actions. Yeast and fungal cell wall components contain pathogenassociated molecular patterns, which interact with toll-like receptors and which activate the innate immune system (Heine and Ulmer, 2005). The GI epithelial cells express all of the toll-like receptors (Harris et al., 2006). Hence, it is possible that the product restores neutrophil markers of innate immunity via toll-like receptor-2 and -4 activation in the GI tract.

An unexpected observation in the first study was the improvement in faecal consistency caused by the administration of DEX. The cause for this is not known. DEX is typically administered as an anti-inflammatory. A search of the human medical literature did not reveal any associations or side effects that might have predicted or explained this response.

A caveat to this experiment and to experiment 2 is that the neutrophil preparation that we generated, although very pure, contained contaminating cell types. It is possible that a portion of the effects that we have attributed to neutrophils could also have arisen within another cell population.

\section{Experiment 2}

Goals of the second experiment were to re-investigate the ability of OmniGen-AF to increase expression of neutrophil L-selectin, to determine whether the properties of the additive were affected by pelleting (i.e. heating) the feed and to examine abilities of other feed products (DTX, MTB100 and Nutrasound-Pak) to bring about changes in neutrophil L-selectin abundance to those noted in experiment 1. We also evaluated effects of OmniGen-AF on circulating concentrations of neutrophils and lymphocytes.

Animals that were fed pelleted concentrate portions of the rations gained weight more rapidly than animals that received concentrate supplements in a mash form. Animals were provided the same amount of concentrate whether it was provided in a mash or pelleted form; hence, we propose that the stimulation in gain was due to the increased intake of free-choice forages in pellet-fed animals. Forage intake was not monitored in this study; hence, we do not know if forage intake may have been affected by the treatments.

OmniGen-AF increased abundance of neutrophil L-selectin protein several-fold in animals irrespective of whether it was fed as a mash or in a pellet (i.e. heated). The ability to modify the expression of neutrophil L-selectin was unaffected by sex (wethers or ewe lambs). Other products tested in this experiment were unable to bring about changes in the expression of L-selectin. OmniGen-AF also increased circulating levels of both neutrophils and lymphocytes. Mechanisms for these changes were not assessed, but we propose that the feed additive either enhanced the formation of mature leukocytes (e.g. via increased circulating levels of IL-1 $\beta$ ) or via delayed apoptosis.

\section{Conclusion}

We examined the ability of a commercially available feed additive (OmniGen-AF) to alter concentrations of markers of innate immunity in neutrophils of immunosuppressed sheep. Concentrations of neutrophil L-selectin were increased by the product. Further, effects on neutrophil IL-1 $\beta$ abundance were also noted, but were dependent on the presence of a pathogen (mould) in the ration. Other feed additives lacked the ability to alter abundance of neutrophil innate immune markers. We conclude the product has the potential to alter innate immune function and that its effects are manifest in the neutrophil. 


\section{Acknowledgements}

We are grateful to Dr Rob Bildfell (Oregon State University College of Veterinary Medicine; OSUCVM) who performed necropsies on animals and provided veterinary support for the project. We are also grateful to Dr Russell Crisman (OSUCVM) who provided guidelines on withdrawal times for DEX-treated sheep. Finally, we appreciate the support of the OSU Sheep Center, to its manager (Tom Nichols) and to Dr James Males (Head, OSU Department of Animal Sciences) for support during the project.

\section{References}

Brent R, Kingston RE, Seidman JG, Struhl K and Ausubel FM 2003. Current protocols in molecular biology. John Wiley and Sons, New York.

Burton JL and Kehrli Jr ME 1995. Regulation of neutrophil adhesion molecules and shedding of Staphylococcus aureus in milk of cortisol- and dexamethasone-treated cows. American Journal of Veterinary Research 56, 997-1006.

Burton JL and Erskine RJ 2003. Immunity and mastitis. Some new ideas for an old disease. Veterinary Clinics of North American Food Animal Practice 19, $1-45$.

Burton JL, Kehrli Jr ME, Kapil S and Horst RL 1995. Regulation of L-selectin and CD18 on bovine neutrophils by glucocorticoids: effects or cortisol and dexamethasone. Journal of Leukocyte Biology 57, 317-325.

Burton JL, Madsen SA, Chang LC, Weber PS, Buckham KR, van Dorp R, Hickey $M C$ and Earley B 2005. Gene expression signatures in neutrophils exposed to glucocorticoids: a new paradigm to help explain 'neutrophil dysfunction' in parturient dairy cows. Veterinary Immunology and Immunopathology 105, 197-219.
Harris G, KuoLee R and Chen W 2006. Role of Toll-like receptors in health and diseases of gastrointestinal tract. World Journal of Gastroenterology 12 2149-2160.

Heine $\mathrm{H}$ and Ulmer AJ 2005. Recognition of bacterial products by toll-like receptors. Chemical and. Immunological Allergy 86, 99-119.

Janeway CA, Travers P, Walport M and Shlomchik MJ 2005. Immunobiology. The immune system in health and disease. Garland Science, New York.

Madsen SA, Chang LC, Hickey MC, Rosa GJ, Coussens PM and Burton JL 2004. Microarray analysis of gene expression in blood neutrophils of parturient cows. Physiological Genomics 16, 212-221.

Mallard BA, Dekkers JC, Ireland MJ, Leslie KE, Sharif S, Vankampen CL, Wagter $\mathrm{L}$ and Wilkie $\mathrm{BN}$ 1998. Alteration in immune responsiveness during the peripartum period and its ramification on dairy cow and calf health. Journal of Dairy Science 81, 585-595.

Puntenney SB, Wang YQ and Forsberg NE 2003. Mycotic infections in livestock: recent insights and studies on etiology, diagnostics and prevention of hemorrhagic bowel syndrome. Proceedings of the Southwest Animal Nutrition Conference, pp. 49-63.

Statistical Analysis Systems Institute 2005. SAS/STAT software, version 5.0. SAS Institute, Cary, NC.

Steel RGD and Torrie JH 1980. Principles and procedures of statistics. McGrawHill Book Co., New York.

Weber PS, Madsen SA, Smith GW, Ireland JJ and Burton JL 2001. Pretranslational regulation of neutrophil L-selectin in glucocorticoid-challenged cattle. Veterinary Immunology and Immunopathology 83, 213-240.

Weber PS, Toelboell T, Chang LC, Tirrell JD, Saama PM, Smith GW and Burton JL 2004. Mechanisms of glucocorticoid-induced down-regulation of neutrophil L-selectin in cattle: evidence for effects at the gene-expression level and primarily on blood neutrophils. Journal of Leukocyte Biology 75, 815-827. 